\title{
Note
}

\section{Synthesis of a repeating pentasaccharide fragment of the capsular polysaccharide of Streptococcus pneumoniae type $18 \mathrm{C}$}

\author{
Augusta M.P. van Steijn, Jos G.M. van der Ven, Paul van Seeventer, \\ Johannis P. Kamerling, and Johannes F.G. Vliegenthart \\ Bijvoet Center, Department of Bio-Organic Chemistry, Utrecht University, P.O. Box 80.075, \\ NL-3508 TB Utrecht (Netherlands)
}

(Received August 28th, 1991; accepted October 11th, 1991)

In the framework of our investigations on the development of synthetic vaccines $^{1}$, based on oligosaccharide conjugates, against infections by Streptococcus pneumoniae serotypes, attention has been focused on the preparation of structural elements of the capsular polysaccharide of serotype $18 \mathrm{C}^{2}(1)$, being one of the constituents of Pneumovax ${ }^{\mathscr{O}} 23$. Recently, we have carried out ${ }^{3}$ the synthesis of the non-glycerol-phosphorylated elements $\beta$-D-Gal $p-(1 \rightarrow 4)-\alpha$-D-Glcp- $(1 \rightarrow 3)-\alpha-\mathrm{L}-$ Rhap-OMe and $\alpha$-D-Glcp-( $1 \rightarrow 2)-[\beta$-D-Glc $p-(1 \rightarrow 4)]-\beta$-D-Gal $p$-OMe, and the glycerol-phosphorylated elements $s n$-Glycerol-(3-P $\rightarrow 3$ )- $\beta$-D-Gal $p$-(1 $\rightarrow 4)-\alpha$-DGlc $p-(1 \rightarrow 3)-\alpha-\mathrm{L}-\mathrm{Rha} p-\mathrm{OMe}$ and $\alpha$-D-Glcp-(1 $\rightarrow 2)$-[sn-Glycerol-(3-P $\rightarrow 3)]-[\beta$-DGlc $p$ - $(1 \rightarrow 4)]-\beta$-D-Galp-OMe.

$$
\alpha \text {-D-Glcp- }(1 \rightarrow 2)
$$

$[\rightarrow 4)-\beta$-D-Glcp-(1 $\rightarrow 4)-\beta$-D-Gal $p-(1 \rightarrow 4)-\alpha$-D-Glc $p-(1 \rightarrow 3)-\alpha$-L-Rhap-(1 $\rightarrow]_{n}$

Glycerol-1-phosphate $\rightarrow 3)^{\prime} \quad 1 \quad(+1$ OAc)

For the synthesis of the non-glycerol-phosphorylated pentasaccharide repeating unit, $\quad \alpha$-D-Glc $p$ - $(1 \rightarrow 2)-[\beta$-D-Glcp- $(1 \rightarrow 4)]-\beta$-D-Gal $p-(1 \rightarrow 4)-\alpha$-D-Glc $p-(1 \rightarrow 3)-\mathrm{L}-$ Rhap $(2 \alpha \beta)$, of the capsular polysaccharide of $S$. pneumoniae type $18 \mathrm{C}$, the trisaccharide derivative benzyl 2,4-di- $O$-benzyl-3-O-[2,3,6-tri- $O$-benzyl-4- $O-(2,4,6-$ tri- $O$-acetyl-3- $O$-allyl- $\beta$-D-galactopyranosyl)- $\alpha$-D-glucopyranosyl]- $\alpha-\mathrm{L}$-rhamnopyran$\operatorname{oside}^{3}$ (3) was used as a key intermediate. This intermediate offers also the possibility to prepare the glycerol-phosphate-containing analogue of 2.

Deacetylation of $3(\rightarrow 4)$, followed by benzylidenation afforded 5 ( $77 \%$ from 3 ). Coupling of 5 with ethyl 2,3,4,6-tetra-O-benzyl-1-thio- $\beta$-D-glucopyranoside ${ }^{4}$ in ether

Correspondence to: Dr. J.P. Kamerling, Bijvoet Center, Department of Bio-Organic Chemistry, Utrecht University, P.O. Box 80.075 , NL-3508 TB Utrecht, The Netherlands. 
TABLE I

500-MHz ${ }^{1} \mathrm{H}-\mathrm{NMR}$ data ${ }^{a}$ for pentasaccharide 2

\begin{tabular}{|c|c|c|c|c|c|c|c|}
\hline \multirow[t]{2}{*}{ Proton/ $J$} & \multicolumn{7}{|c|}{$\delta(\mathrm{ppm}) / J(\mathrm{~Hz})$} \\
\hline & $\alpha$-Rha & $\beta$-Rha & $\alpha-\mathrm{Glc}^{\prime}(\alpha)^{b}$ & $\alpha-\operatorname{Glc}^{\prime}(\beta)^{b}$ & $\beta-\mathrm{Gal}^{\prime \prime}$ & $\alpha-\mathrm{Glc}^{\prime \prime}$ & $\beta-\mathrm{Glc}^{\prime \prime \prime}$ \\
\hline H-1 & 5.153 & 4.863 & 5.081 & 5.107 & $4.655 / 2^{\circ}$ & 5.338 & 4.672 \\
\hline H-2 & 4.126 & 4.147 & 3.625 & 3.625 & 3.758 & 3.519 & 3.355 \\
\hline H-3 & 3.861 & 3.683 & 3.939 & 3.941 & 3.864 & 3.733 & 3.507 \\
\hline H-4 & 3.546 & 3.476 & 3.811 & 3.803 & 4.189 & 3.443 & 3.423 \\
\hline $\mathrm{H}-5$ & $3.906^{d}$ & $3.453^{d}$ & 4.128 & 4.110 & n.d. & 4.083 & 3.42 \\
\hline $\mathrm{CH}_{3}$ & 1.292 & 1.309 & - & - & - & - & - \\
\hline$J_{1,2}$ & 1.8 & $\sim 0$ & 3.8 & 3.8 & 8.1 & 3.9 & 8.1 \\
\hline
\end{tabular}

${ }^{a}$ Chemical shifts are relative to the signal of internal acetone $\left(\delta 2.225 \mathrm{ppm}\right.$ in $\left.\mathrm{D}_{2} \mathrm{O}\right){ }^{b}$ Doubling of the $\alpha$-Glc' series is due to the anomers of Rha. ${ }^{c}$ Two chemical shift values due to the anomers of $\mathbf{R h a}$. ${ }^{d}$ Assignment of the anomeric configuration is also based on the $\delta$ values of Rha H-5 (ref. 11).

with methyl triflate ${ }^{5}$ as the promoter yielded the tetrasaccharide derivatives $6 \alpha$ (50\%) and $6 \beta(26 \%)$, having the additional glucosyl unit in $\alpha$ - and $\beta$-(1 $\rightarrow 2)$ linkages, respectively. Selective opening of the 4,6-O-benzylidene ring of $6 \alpha$, using sodium cyanoborohydride-hydrochloric acid in tetrahydrofuran ${ }^{6}$, gave the corresponding 6-benzyl ether $(7,51 \%)$. Condensation of 7 with 2,3,4,6-tetra- $O$-acetyl- $\alpha$ D-glucopyranosyl bromide in dichloromethane-toluene, using silver triflate as the catalyst at $-25^{\circ}$, gave $8(77 \%)$. Deallylation ${ }^{7}$ of $8(\rightarrow 9,61 \%)$, then deacetylation, and debenzylation afforded the target compound $2 \alpha \beta(91 \%)$. The ${ }^{1} \mathrm{H}-\mathrm{NMR}$ data of 2, obtained by $2 \mathrm{D}$ COSY and 2D HOHAHA measurements, are presented in Table I.
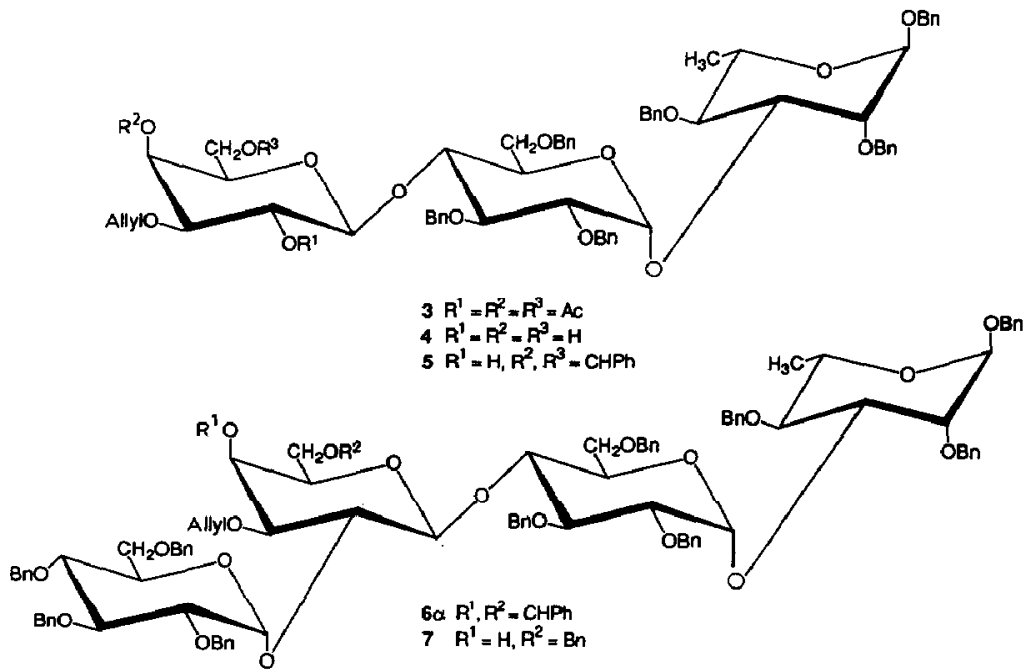


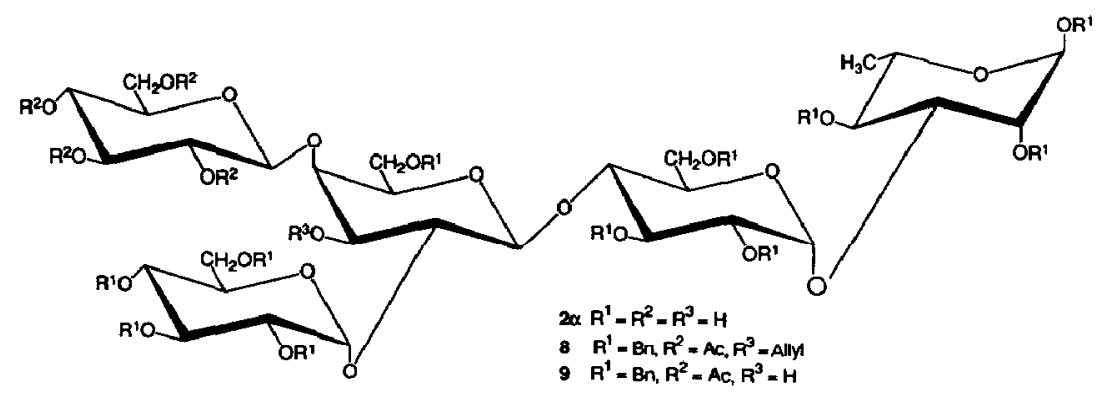

EXPERIMENTAL

General methods. $-{ }^{1} \mathrm{H}-\mathrm{NMR}$ spectra $(360$ and $500 \mathrm{MHz})$ were recorded at $25^{\circ}$ with a Bruker HX 360 or AM 500 spectrometer (Bijvoet Center, Utrecht University). 2D Double-quantum-filtered ${ }^{1} \mathrm{H}-{ }^{1} \mathrm{H}$ correlation spectra (2D DQF ${ }^{1} \mathrm{H}-{ }^{1} \mathrm{H}$ COSY) were recorded in the phase-sensitive mode ${ }^{8}$, and $2 \mathrm{D}$ homonuclear Hartmann-Hahn spectra (2D HOHAHA) with a MLEV-17 mixing sequence of $120 \mathrm{~ms}$ (ref. 9). ${ }^{13} \mathrm{C}$-NMR spectra (APT, $50 \mathrm{MHz}$ ) were recorded at $25^{\circ}$ with a Bruker WP 200 spectrometer. Chemical shifts $(\delta)$ are given in ppm relative to the signal for internal $\mathrm{Me}_{4} \mathrm{Si}\left(\mathrm{CDCl}_{3}\right)$ or sodium 4,4-dimethyl-4-silapentane-1-sulfonate $\left(\mathrm{D}_{2} \mathrm{O}\right.$; indirectly to internal acetone, $\delta 2.225$ ) for ${ }^{1} \mathrm{H}$, and to the signal for internal $\mathrm{Me}_{4} \mathrm{Si}$ $\left(\mathrm{CDCl}_{3}\right.$; indircetly to $\left.\mathrm{CDCl}_{3}, \delta 76.9\right)$ or external $\mathrm{Me}_{4} \mathrm{Si}\left(\mathrm{D}_{2} \mathrm{O}\right.$; indirectly to internal acetone, $\delta 31.55$ ) for ${ }^{13} \mathrm{C}$.

Column chromatography was performed on Kieselgel 60 (Merck, < 230 mesh) and fractions were monitored by TLC on Kieselgel $60 \mathrm{~F}_{254}$ (Merck). Detection was effected by charring with $\mathrm{H}_{2} \mathrm{SO}_{4}$ after examination under UV light. Optical rotations were measured at $20^{\circ}$ with a Perkin-Elmer 241 polarimeter, using a $10-\mathrm{cm} 1-\mathrm{mL}$ cell. In the workup procedures, washings were carried out three times with appropriate quantities of $\mathrm{H}_{2} \mathrm{O}$ or aq $5 \% \mathrm{NaHCO}_{3}$ unless indicated otherwise. Solvents were evaporated under reduced pressure at $40^{\circ}$ (bath). All solvents were distilled from appropriate drying agents.

Because of hygroscopicity, satisfactory elemental analyses of $2 \alpha \beta, 8$, and 9 could not be obtaincd.

Benzyl 3-O-[4-O-(3-O-allyl-4,6-O-benzylidene- $\beta$-D-galactopyranosyl)-2,3,6-tri-O-

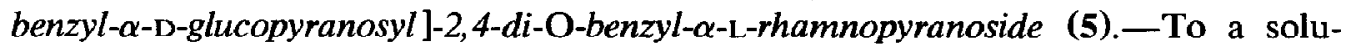
tion of benzyl 2,4-di- $O$-benzyl-3- $O$-[2,3,6-tri- $O$-benzyl-4- $O$-(2,4,6-tri- $O$-acetyl-3- $O$ allyl- $\beta$-D-galactopyranosyl)- $\alpha$-D-glucopyranosyl]- $\alpha$-L-rhamnopyranoside ${ }^{3}$ (3; 349 mg, $0.33 \mathrm{mmol})$ in $\mathrm{MeOH}(10 \mathrm{~mL})$ was added sodium methoxide to $\mathrm{pH} 10$. After stirring for $48 \mathrm{~h}$, the mixture was neutralised with Dowex-50 $\left(\mathrm{H}^{+}\right)$resin, filtered, and concentrated. Column chromatography ( $75: 25 \mathrm{CH}_{2} \mathrm{Cl}_{2}-\mathrm{EtOAc}$ ) of the residue gave 4 , isolated as a syrup. To a solution of 4 in $N, N$-dimethylformamide ( $1 \mathrm{~mL}$ ) and $\alpha, \alpha$-dimethoxytoluene ( $3 \mathrm{~mL}$ ) was added $p$-toluenesulfonic acid (25 $\mathrm{mg}$ ). The 
solution was stirred overnight (TLC, 95:5 $\mathrm{CH}_{2} \mathrm{Cl}_{2}$-EtOAc; $5 R_{\mathbf{F}} \mathbf{0 . 3 0}$ ), diluted with $\mathrm{CH}_{2} \mathrm{Cl}_{2}(400 \mathrm{~mL})$, washed with aq $5 \% \mathrm{NaHCO}_{3}$ and $\mathrm{H}_{2} \mathrm{O}$, dried $\left(\mathrm{Na}_{2} \mathrm{SO}_{4}\right)$, filtered, and concentrated. Column chromatography of the residue afforded 5 , isolated as a glass $(289 \mathrm{mg}, 77 \%),[\alpha]_{\mathrm{D}}+17^{\circ}\left(c 1, \mathrm{CHCl}_{3}\right)$. NMR data $\left(\mathrm{CDCl}_{3}\right)$ : ${ }^{13} \mathrm{C}, \delta$ 139.1-137.3 and $128.6-126.3\left(C_{6} \mathrm{H}_{5} \mathrm{CH}_{2} \mathrm{O}\right.$ and $\left.\mathrm{C}_{6} \mathrm{H}_{5} \mathrm{CH}\right), \quad 135.0$ $\left(\mathrm{H}_{2} \mathrm{C}=\mathrm{CHCH}_{2} \mathrm{O}\right), 117.1\left(\mathrm{H}_{2} \mathrm{C}=\mathrm{CHCH}_{2} \mathrm{O}\right), 103.6,100.9\left(\mathrm{C}^{\prime \prime}\right.$ and $\left.\mathrm{PhCH}\right), 97.2$, $95.0\left(\mathrm{C}-1,1^{\prime}\right), 17.8(\mathrm{C}-6) ;{ }^{1} \mathrm{H}, \delta 7.412-7.166(\mathrm{~m}, 35 \mathrm{H}, 7 \mathrm{Ph}), 5.459(\mathrm{~m}, 1 \mathrm{H}$, $\left.\mathrm{H}_{2} \mathrm{C}=\mathrm{CHCH}_{2} \mathrm{O}\right), 5.456(\mathrm{~s}, 1 \mathrm{H}, \mathrm{PhCH}), 5.309$ and $5.197(2 \mathrm{~m}$, each $1 \mathrm{H}$, $\mathrm{H}_{2} \mathrm{C}=\mathrm{CHCH}_{2} \mathrm{O}$ ), 5.131 (d, $\left.1 \mathrm{H}, \mathrm{H}-1^{\prime}\right), 1.358(\mathrm{~d}, 3 \mathrm{H}, \mathrm{H}-6,6,6), J_{1^{\prime}, 2^{\prime}}, 3.5, J_{5,6} 6.1 \mathrm{~Hz}$.

Anal. Calcd for $\mathrm{C}_{70} \mathrm{H}_{76} \mathrm{O}_{15}: \mathrm{C}, 72.64 ; \mathrm{H}, 6.62$. Found: C, 72.28; H, 6.62.

Benzyl 3-O-\{4-O-[3-O-allyl-4,6-O-benzylidene-2-O-(2,3,4,6-tetra-O-benzyl- $\alpha, \beta-\mathrm{D}-$ glucopyranosyl )- $\beta$-D-galactopyranosyl ]-2,3,6-tri-O-benzyl- $\alpha$-D-glucopyranosyl $\}-2,4-d i$ O-benzyl- $\alpha$-L-rhamnopyranoside $(6 \alpha \beta)$. - To a solution of $5(537 \mathrm{mg}, 0.46 \mathrm{mmol})$ and ethyl 2,3,4,6-tetra- $O$-benzyl-1-thio- $\beta$-D-glucopyranoside ${ }^{4}(542 \mathrm{mg}, 0.93 \mathrm{mmol}$ ) in ether $(20 \mathrm{~mL})$, containing powdered $4 \mathrm{~A}$ molecular sieves $(1.5 \mathrm{~g})$, was added methyl triflate ${ }^{5}(0.5 \mathrm{~mL}, 4.56 \mathrm{mmol})$, and the mixture was stirred for $16 \mathrm{~h}$. Triethylamine $(2 \mathrm{~mL})$ was added and, after $5 \mathrm{~min}$, the mixture was diluted with $\mathrm{CH}_{2} \mathrm{Cl}_{2}(250 \mathrm{~mL})$, filtered through Celite, washed with $\mathrm{H}_{2} \mathrm{O}$, dried $\left(\mathrm{Na}_{2} \mathrm{SO}_{4}\right)$, filtered, and concentrated. Column chromatography $[7: 3$ light petroleum (bp $40-60^{\circ}$ )-EtOAc] of the residue gave $6 \alpha$, isolated as a white glass (391 $\mathrm{mg}, 50 \%$ ), $[\alpha]_{\mathrm{D}}+52^{\circ}\left(c 1, \mathrm{CHCl}_{3}\right), R_{\mathrm{F}} 0.41$; and $6 \beta$, isolated as a white glass (205 $\left.\mathrm{mg}, 26 \%\right)$, $[\alpha]_{\mathrm{D}}+21^{\circ}\left(c 1, \mathrm{CHCl}_{3}\right), R_{\mathrm{F}}$ 0.64. NMR data $\left(\mathrm{CDCl}_{3}\right): 6 \alpha{ }^{13} \mathrm{C}, \delta 139.0-137.2$ and 128.9-126.4 $\left(\mathrm{C}_{6} \mathrm{H}_{5} \mathrm{CH}_{2} \mathrm{O}\right.$ and $\left.\mathrm{C}_{6} \mathrm{H}_{5} \mathrm{CH}\right), \quad 134.9 \quad\left(\mathrm{H}_{2} \mathrm{C}=\mathrm{CHCH}_{2} \mathrm{O}\right), \quad 117.1$ $\left(\mathrm{H}_{2} \mathrm{C}=\mathrm{CHCH}_{2} \mathrm{O}\right), 102.1,100.9\left(\mathrm{C}-1^{\prime \prime}\right.$ and $\left.\mathrm{PhCH}\right), 97.2,96.0$, and $95.1\left(\mathrm{C}-1,1^{\prime}, 1^{\prime \prime \prime}\right)$, $18.0(\mathrm{C}-6) ;{ }^{1} \mathrm{H}, \delta 7.443-7.055(\mathrm{~m}, 55 \mathrm{H}, 11 \mathrm{Ph}), 5.956\left(\mathrm{~m}, 1 \mathrm{H}, \mathrm{H}_{2} \mathrm{C}=\mathrm{CHCH}_{2} \mathrm{O}\right)$, 5.471 (s, $1 \mathrm{H}, \mathrm{PhCH}$ ), 5.312 (d, $\left.1 \mathrm{H}, \mathrm{H}-1^{\prime \prime \prime}\right), 5.144$ (d, $\left.1 \mathrm{H}, \mathrm{H}-1^{\prime}\right), 5.255$ and 5.076 (2 $\mathrm{m}$, each $\left.1 \mathrm{H}, \mathrm{H}_{2} \mathrm{C}=\mathrm{CHCH}_{2} \mathrm{O}\right), 1.228(\mathrm{~d}, 3 \mathrm{H}, \mathrm{H}-6,6,6), J_{5,6} 6.2, J_{1^{\prime}, 2^{\prime}} 3.8, J_{1^{\prime \prime \prime}, 2^{\prime \prime \prime}} 3.4$ $\mathrm{Hz} ; 6 \beta{ }^{13} \mathrm{C}, \delta 139.4-137.3$ and $129.5-126.5\left(C_{6} \mathrm{H}_{5} \mathrm{CH}_{2} \mathrm{O}\right.$ and $\left.C_{6} \mathrm{H}_{5} \mathrm{CH}\right), 134.9$ $\left(\mathrm{H}_{2} \mathrm{C}=\mathrm{CHCH} \mathrm{HCH}_{2} \mathrm{O}\right), 117.0\left(\mathrm{H}_{2} \mathrm{C}=\mathrm{CHCH}_{2} \mathrm{O}\right), 102.4,102.0$, and $101.3\left(\mathrm{C}-1^{\prime \prime}, 1^{\prime \prime \prime}\right.$ and $\mathrm{PhCH})$, 97.3, 96.1 (C-1,1'), 17.8 (C-6).

Anal. Calcd for $\mathrm{C}_{104} \mathrm{H}_{110} \mathrm{O}_{20}: \mathrm{C}, 74.35 ; \mathrm{H}, 6.60$. Found $6 \alpha: \mathrm{C}, 74.13 ; \mathrm{H}, 6.88$. Found $6 \beta$ : C, 74.19; $\mathrm{H}, 6.97$.

Benzyl 3-O-\{4-O-[3-O-allyl-6-O-benzyl-2-O-(2,3,4,6-tetra-O-benzyl- $\alpha-\mathrm{D}-$ glucopyranosyl)- $\beta$-D-galactopyranosyl ]-2,3,6-tri-O-benzyl- $\alpha$-D-glucopyranosyl $\}-2,4-d i-\mathrm{O}-$

benzyl- $\alpha$-L-rhamnopyranoside (7). - To a solution of $6 \alpha(360 \mathrm{mg}, 0.21 \mathrm{mmol})$ and sodium cyanoborohydride ${ }^{6}(168 \mathrm{mg}, 2.7 \mathrm{mmol})$ in freshly distilled tetrahydrofuran $(10 \mathrm{~mL})$, containing $3 \mathrm{~A}$ molecular sieves $(1.5 \mathrm{~g})$, was added satd $\mathrm{HCl}$ in ether until the evolution of gas ceased. After $1 \mathrm{~h}$, when TLC [7:3 light petroleum (bp $\left.\left.40-60^{\circ}\right)-E t O A c\right]$ indicated the reaction to be complete, the mixture was diluted with $\mathrm{CH}_{2} \mathrm{Cl}_{2}(250 \mathrm{~mL})$ and $\mathrm{H}_{2} \mathrm{O}(50 \mathrm{~mL})$, filtered through Celite, washed with aq $5 \% \mathrm{NaHCO}_{3}$ and $\mathrm{H}_{2} \mathrm{O}$, dried $\left(\mathrm{Na}_{2} \mathrm{SO}_{4}\right)$, filtered, and concentrated. Column chromatography of the residue gave 7 , isolated as a syrup $(183 \mathrm{mg}, 51 \%),[\alpha]_{\mathrm{D}}$ $+45^{\circ}\left(c 1, \mathrm{CHCl}_{3}\right), R_{\mathrm{F}} 0.58 .{ }^{13} \mathrm{C}-\mathrm{NMR}$ data $\left(\mathrm{CDCl}_{3}\right): \delta 138.9-137.2$ and $128.7-$ 
127.2 $\left(\mathrm{C}_{6} \mathrm{H}_{5} \mathrm{CH}_{2} \mathrm{O}\right), 134.0\left(\mathrm{H}_{2} \mathrm{C}=\mathrm{CHCH}_{2} \mathrm{O}\right), 118.0\left(\mathrm{H}_{2} \mathrm{C}=\mathrm{CHCH}_{2} \mathrm{O}\right), 101.7\left(\mathrm{C}-1^{\prime \prime}\right)$, 97.3, 96.1, and $95.0\left(\mathrm{C}-1,1^{\prime}, 1^{\prime \prime \prime}\right), 18.0(\mathrm{C}-6)$.

Anal. Calcd for $\mathrm{C}_{104} \mathrm{H}_{112} \mathrm{O}_{20}$ : C, 74.26; $\mathrm{H}, 6.71$. Found: $\mathrm{C}, 74.59 ; \mathrm{H}, 6.72$.

Benzyl 3-O-\{4-O-[3-O-allyl-6-O-benzyl-4-O-(2,3,4,6-tetra-O-acetyl- $\beta$-D-glucopyranosyl)-2-O-(2,3,4,6-tetra-O-benzyl- $\alpha$-D-glucopyranosyl)- $\beta$-D-galactopyranosyl $] 2$, 3,6-tri-O-benzyl- $\alpha$-D-glucopyranosyl\}-2,4-di-O-benzyl- $\alpha$-L-rhamnopyranoside (8).-A solution of silver triflate $(160 \mathrm{mg}, 0.6 \mathrm{mmol})$ in toluene $(2.5 \mathrm{~mL})$ was added dropwise during $30 \mathrm{~min}$ in the dark to a stirred mixture of $7(132 \mathrm{mg}, 78 \mu \mathrm{mol})$, 2,3,4,6-tetra- $O$-acetyl- $\alpha$-D-glucopyranosyl bromide (117 $\mathrm{mg}, 0.29 \mathrm{mmol}$ ), and powdered $4 \mathrm{~A}$ molecular sieves $(500 \mathrm{mg})$ in $\mathrm{CH}_{2} \mathrm{Cl}_{2}(2 \mathrm{~mL})$ and toluene $(2 \mathrm{~mL})$ at $-40^{\circ}$. The stirring was continued for $2.5 \mathrm{~h}$ at $-25^{\circ}$, until TLC $(6: 1$ tolueneacetone) showed the absence of $7\left(R_{\mathrm{F}} 0.74\right)$. Pyridine was added, and the mixture was diluted with $\mathrm{CH}_{2} \mathrm{Cl}_{2}(150 \mathrm{~mL})$, filtered through Celite, washed with aq $10 \%$ sodium thiosulfate $(2 \times 25 \mathrm{~mL})$ and $\mathrm{H}_{2} \mathrm{O}$, dried $\left(\mathrm{Na}_{2} \mathrm{SO}_{4}\right)$, filtered, and concentrated. Column chromatography of the residue afforded 8 , isolated as a glass (122 $\mathrm{mg}, 77 \%),[\alpha]_{\mathrm{D}}+32^{\circ}\left(c 1, \mathrm{CHCl}_{3}\right), R_{\mathrm{F}}$ 0.59. NMR data $\left(\mathrm{CDCl}_{3}\right):{ }^{13} \mathrm{C}, \delta 170.5$, 170.2, 169.3, and 168.8 $\left(4 \mathrm{COCH}_{3}\right), 138.9-137.2$ and $128.9-127.3\left(C_{6} \mathrm{H}_{5} \mathrm{CH}_{2} \mathrm{O}\right)$, $134.1\left(\mathrm{H}_{2} \mathrm{C}=\mathrm{CHCH}_{2} \mathrm{O}\right), 117.5\left(\mathrm{H}_{2} \mathrm{C}=\mathrm{CHCH}_{2} \mathrm{O}\right), 102.2,99.2,97.4,97.0$, and 95.5 $\left(\mathrm{C}-1,1^{\prime}, 1^{\prime \prime}, 1^{\prime \prime \prime}, 1^{\prime \prime \prime}\right), 20.5-20.4\left(\mathrm{COCH}_{3}\right), 18.0(\mathrm{C}-6) ;{ }^{1} \mathrm{H}, \delta$ 7.330-7.121 (m, $55 \mathrm{H}, 11$ $\mathrm{Ph}), 5.929\left(\mathrm{~m}, 1 \mathrm{H}, \mathrm{H}_{2} \mathrm{C}=\mathrm{CHCH}_{2} \mathrm{O}\right), 2.028,1.963$, and $1.893(3 \mathrm{~s}, 6,3,3 \mathrm{H}, 4 \mathrm{Ac})$, $1.281(\mathrm{~d}, 3 \mathrm{H}, \mathrm{H}-6,6,6), J_{5,6} 6.2 \mathrm{~Hz}$.

Benzyl 2,4-di-O-benzyl-3-O-\{4-O-[6-O-benzyl-4-O-(2,3,4,6-tetra-O-acetyl- $\beta$-Dglucopyranosyl)-2-O-(2,3,4,6-tetra-O-benzyl- $\alpha$-D-glucopyranosyl)- $\beta$-D-galactopyranosyl]-2,3,6-tri-O-benzyl- $\alpha$-D-glucopyranosyl $\}-\alpha$-L-rhamnopyranoside (9).-A solution of $8(25 \mathrm{mg}, 12.4 \mu \mathrm{mol})$, tris(triphenylphosphine)rhodium(I) chloride $(15 \mathrm{mg}, 16$ $\mu \mathrm{mol}$ ), and 1,4-diazobicyclo[2.2.2]octane (5 mg, $45 \mu \mathrm{mol})$ in $7: 3: 1 \mathrm{EtOH}-$ benzene- $\mathrm{H}_{2} \mathrm{O}(3 \mathrm{~mL})$ was boiled under reflux ${ }^{7}$ for $16 \mathrm{~h}$, then concentrated. The residue was dissolved in 95:5 acetone- $\mathrm{H}_{2} \mathrm{O}(4 \mathrm{~mL})$ containing mercury(II) oxide ( $5 \mathrm{mg}, 23 \mu \mathrm{mol}$ ) and mercury(II) chloride ( $20 \mathrm{mg}, 74 \mu \mathrm{mol}$ ), and the solution was stirred for $1.5 \mathrm{~h}$ at room temperature. When TLC [6:4 light petroleum (bp 40-60 $0^{\circ}$-EtOAc] indicated complete conversion into $9\left(R_{\mathrm{F}} 0.58\right)$, the mixture was concentrated and then dissolved in $\mathrm{CH}_{2} \mathrm{Cl}_{2}(100 \mathrm{~mL})$. The solution was washed with aq $30 \% \mathrm{KBr}(3 \times 15 \mathrm{~mL})$ and $\mathrm{H}_{2} \mathrm{O}$, dried $\left(\mathrm{Na}_{2} \mathrm{SO}_{4}\right)$, filtered, and concentrated. Column chromatography of the residue afforded 9 , isolated as a syrup (15 $\mathrm{mg}, 61 \%),[\alpha]_{\mathrm{D}}+57^{\circ}\left(c 1, \mathrm{CHCl}_{3}\right) .{ }^{1} \mathrm{H}-\mathrm{NMR}$ data $\left(\mathrm{CDCl}_{3}\right): \delta 7.377-7.085(\mathrm{~m}, 55$ $\mathrm{H}, 11 \mathrm{Ph}), 2.072,2.024,1.973$, and $1.760(4 \mathrm{~s}$, each $1 \mathrm{H}, 4 \mathrm{Ac}), 1.196(\mathrm{~d}, 3 \mathrm{H}$, H-6,6,6).

3-O-[4-O-(2-O- $\alpha$-D-Glucopyranosyl-4-O- $\beta$-D-glucopyranosyl- $\beta$-D-galactopyranosyl)- $\alpha$-D-glucopyranosyll- $\alpha, \beta$-L-rhamnopyranose $(2 \alpha \beta)$. - To a solution of $9(25 \mathrm{mg}$, $13 \mu \mathrm{mol}$ ) in $\mathrm{MeOH}(5 \mathrm{~mL})$ was added sodium methoxide to $\mathrm{pH} 10$. After $16 \mathrm{~h}$, the mixture was neutralised with Dowex-50 $\left(\mathrm{H}^{+}\right)$resin, filtered, and concentrated. Column chromatography $\left(R_{\mathrm{F}} 0.46,85: 10: 5 \mathrm{CH}_{2} \mathrm{Cl}_{2}\right.$-acetone-MeOH) of the residue afforded deacetylated 9 , isolated as a syrup. To a solution of the residue in 
3:2 MeOH-2-propanol (5 mL) containing a few drops of acetic acid was added $10 \% \mathrm{Pd}-\mathrm{C}(15 \mathrm{mg})$, and the mixture was hydrogenolysed at $4 \mathrm{~kg} / \mathrm{cm}^{2}$ for $16 \mathrm{~h}$, filtered, and concentrated to give $2 \alpha \beta$, isolated as a white powder $(9.3 \mathrm{mg}, 91 \%)$, $[\alpha]_{\mathrm{D}}+77^{\circ}\left(c 1, \mathrm{H}_{2} \mathrm{O}\right) .{ }^{13} \mathrm{C}-\mathrm{NMR}$ data $\left(\mathrm{D}_{2} \mathrm{O}\right): \delta 105.3(161 \mathrm{~Hz})$ and $102.9(163 \mathrm{~Hz})$ $\left(\mathrm{C}-1^{\prime \prime}, 1^{\prime \prime \prime \prime}\right), 99.1(172 \mathrm{~Hz})\left(\mathrm{C}-1^{\prime \prime \prime}\right), 96.3(172 \mathrm{~Hz})\left(\mathrm{C}-1^{\prime}, \alpha\right.$-Rha anomer), $96.1(170 \mathrm{~Hz})$ $\left(\mathrm{C}-1^{\prime}, \beta\right.$-Rha anomer $), 94.9(169 \mathrm{~Hz})(\mathrm{C}-1 \alpha)^{10}, 94.8(156 \mathrm{~Hz})(\mathrm{C}-1 \beta)^{10}, 62.3,62.0$, 61.5 , and $61.0\left(\mathrm{C}-6^{\prime}, 6^{\prime \prime}, 6^{\prime \prime \prime}, 6^{\prime \prime \prime \prime}\right), 18.2(\mathrm{C}-6)$. For the ${ }^{1} \mathrm{H}-\mathrm{NMR}$ data, see Table I.

\section{ACKNOWLEDGMENTS}

This investigation was supported by the Netherlands Foundation for Chemical Research (SON/NWO), the Institute of Molecular Biology and Medical Biotechnology (IMB, Utrecht University), and the Netherlands Innovation Directed Programme for Biotechnology (IOP-b). We thank Dr. B.R. Leeflang for recording the 2D ${ }^{1} \mathrm{H}-\mathrm{NMR}$ spectra.

\section{REFERENCES}

1 J.E.G. van Dam, A. Fleer, and H. Snippe, Antonie van Leeuwenhoek; J. Microbiol. Serol, 58 (1990) 1-47.

2 C. Lugowski and H.J. Jennings, Carbohydr. Res., 131 (1984) 119-129.

3 A.M.P. van Steijn, J.P. Kamerling, and J.F.G. Vliegenthart, Carbohydr. Res., 211 (1991) 261-277.

4 F. Weygand and H. Zeimann, Justus Liebigs Ann. Chem., (1962) 179-198.

5 H. Lönn, J. Carbohydr. Chem., 6 (1987) 301-306.

6 P.J. Garegg, H. Hultberg, and S. Wallin, Carbohydr. Res., 108 (1982) 97-101.

7 E.J. Corey and W.J. Suggs, J. Org. Chem., 38 (1973) 3224.

8 D. Marion and K. Wüthrich, Biochem. Biophys. Res. Commun., 113 (1983) 967-974.

9 A. Bax and D.G. Davis, J. Magn. Reson., 65 (1985) 355-360.

10 K. Bock, C. Pedersen, and H. Pedersen, Adv. Carbohydr. Chem. Biochem., 42 (1984) 193-225.

11 C. Laffite, A.M. Nguygen Phuoc Du, F. Winternitz, R. Wylde, and F. Pratviel-Sosa, Carbohydr. Res., 67 (1978) 91-103. 